\title{
Evaluation of Intraventricular/Intrathecal Antimicrobial Therapy in the Treatment of Nosocomial Meningitis Caused by Multidrug-Resistant Gram-Negative Bacteria after Central Nervous System Surgery
}

\author{
Nagehan Didem Sari $\mathbb{D}^{1},{ }^{1}$ Sevim Baltali $\mathbb{D}^{2},{ }^{2}$ Istemi Serin $\mathbb{D}^{3},{ }^{3}$ and Veysel Antar $\mathbb{D}^{4}$ \\ ${ }^{1}$ University of Health Science, Istanbul Training and Research Hospital, \\ Department of Infectious Diseases and Clinical Microbiology, Istanbul, Turkey \\ ${ }^{2}$ University of Health Science, Istanbul Training and Research Hospital, Department of Anesthesiology and Reanimation, \\ Istanbul, Turkey \\ ${ }^{3}$ University of Health Science, Istanbul Training and Research Hospital, Department of Hematology, Istanbul, Turkey \\ ${ }^{4}$ University of Health Science, Istanbul Training and Research Hospital, Department of Neurological Surgery, Istanbul, Turkey \\ Correspondence should be addressed to Istemi Serin; serinistemi@hotmail.com
}

Received 6 March 2021; Revised 11 August 2021; Accepted 17 August 2021; Published 29 August 2021

Academic Editor: Louis Detolla

Copyright (C) 2021 Nagehan Didem Sari et al. This is an open access article distributed under the Creative Commons Attribution License, which permits unrestricted use, distribution, and reproduction in any medium, provided the original work is properly cited.

\begin{abstract}
Introduction. Postoperative meningitis (POM) is an infection with high mortality and morbidity following central nervous system surgery due to trauma or tumor. Intrathecal/intraventricular (IT/IVT) antibiotic administrations have been considered as the last treatment options for multidrug-resistance (MDR) Gram-negative bacteria that do not respond to intravenous (IV) regimens. IT/ IVT can bypass the blood-brain barrier, obtain a more effective antibiotic concentration in CSF, and reduce systemic side effects. We aimed to determine the characteristics of postoperative patients who were diagnosed with MDR POM during follow-up in our intensive care unit (ICU). Material and Methods. In this study, POM patients who were followed up in ICU after the central nervous system intervention between January 2016 and December 2019 and whose MDR Gram-negative bacteria were isolated from CSF were evaluated. As soon as the patients were diagnosed with POM, a catheter was inserted and treatment was started. Results. Microbiological eradication was achieved in $3 \pm 0.8$ days with $30 \mathrm{mg} /$ day amikacin treatment in POM due to K. pneumoniae and $3.7 \pm 1.95$ days with colistin sodium $10 \mathrm{mg}$ /day treatment in POM due to A. baumannii via IT/IVT catheter. IT/IVT treatment was utilized for a median of 10 days and continued until the defined cure criteria were achieved. While cure was achieved in 6 of 14 POM cases, 8 of them were exitus. Discussion and Conclusion. To avoid the severe consequences of postoperative meningitis, acting fast and adding IT/IVT methods to parenteral administration routes by considering the distribution of MDR microorganisms within the hospital while planning effective treatment will increase the clinical success.
\end{abstract}

\section{Introduction}

Postoperative meningitis (POM) is an infection with high mortality and morbidity following central nervous system surgery due to trauma or tumor with an incidence of $0.8 \%-$ $1.8 \%$ [1-3]. It is mainly caused by cutaneous microorganisms such as coagulase-negative staphylococci, Staphylococcus aureus (S. aureus), and Propionibacterium acnes (P. acnes)
[4]. Gram-negative bacteria also play a role. Antibiotic prophylaxis is recommended for neurosurgery which is mainly directed against Gram-positive bacteria. Nosocomial staphylococci usually carry methicillin resistance. Current guidelines recommend treatment with either an antipseudomonal cephalosporin or an antipseudomonal carbapenem to cover aerobic Gram-negative bacteria and vancomycin to cover staphylococci and $P$. acnes [4]. The choice between a 
cephalosporin and a carbapenem for empirical therapy should be based on the increasing prevalence of broad-spectrum $\beta$-lactamase-producing bacteria. With high prevalence of nosocomial infections caused by carbapenem-resistant Acinetobacter baumannii (A. baumannii) and other Gramnegative bacteria, colistin should be considered in combination with carbapenems until drug sensitivities are determined. The use of colistin alone is not recommended due to its low intrinsic clinical efficacy and poor CSF penetration [4].

Due to the increased drug resistance, the use of different groups of antibiotics, which cannot cross the blood-brain barrier, with different administration routes has become a current issue [5]. Intrathecal/intraventricular (IT/IVT) antibiotic applications come into prominence especially in the treatment of meningitis caused by multidrug-resistant (MDR) Gram-negative bacteria developed after surgical interventions [6-9].

The rate of mortality is higher in patients with Gramnegative bacterial intracranial infections and MDR Gramnegative agents have also more complicated treatment process. A. baumannii, Klebsiella pneumoniae (K. pneumoniae), and Pseudomonas aeruginosa ( $P$. aeruginosa) are the most common MDR Gram-negative agents [10,11]. Against MDR or extensively drug-resistant (XDR) Gram-negative bacteria, there are only a few drugs such as polymyxins and aminoglycosides; however, due to low brain penetration, intracranial infection does not show any improvement even when treated with these antimicrobial agents. Thus, IT or IVT antibiotic administrations have been considered as the last treatment options for MDR/XDR Gram-negative bacteria that do not respond to intravenous (IV) regimens [12]. IT/ IVT can bypass the blood-brain barrier, obtain a more effective antibiotic concentration in CSF, and reduce systemic side effects [12]. Potential neurotoxicity such as chemical meningitis and ventriculitis cannot be ignored, but these reactions have been reported to be mild and also reversible $[10,12]$. The most commonly used IT/IVT agents are aminoglycosides (gentamycin, tobramycin, netilmicin, amikacin, and streptomycin), polymyxins (colistin and polymyxin B), daptomycin, glycopeptides (vancomycin and teicoplanin), tigecycline, and antifungal agents such as amphotericin B and caspofungin [12].

In this article, we aimed to determine the demographic characteristics, laboratory values, and empirical and antimicrobial treatment options in postoperative patients who were diagnosed with MDR POM during follow-up in our intensive care unit (ICU).

\section{Material and Methods}

In this retrospective single-center study, cases of POM who were followed up in Istanbul Training and Research Hospital Intensive Care Unit after the central nervous system intervention between January 2016 and December 2019 and whose MDR Gram-negative bacteria were isolated from CSF were evaluated.

MDR definition was made in accordance with Magiorakos et al. [13]. All patients were administered ceftriaxone or cefazolin during the induction of anesthesia. The definition of nosocomial meningitis/ventriculitis (NM) was based on the classification of the American Centers for Disease Control and Prevention (CDC) [14]. According to the $\mathrm{CDC}$, the case definition is as follows:

(1) $>38^{\circ} \mathrm{C}$ fever, detected 72 hours after the operation

(2) Agent isolation from the CSF

(3) Leukocyte count $>10 / \mathrm{mm}^{3}$, protein $>45 \mathrm{mg} / \mathrm{dL}$, and glucose $<40 \mathrm{mg} / \mathrm{dL}$ in CSF

(4) No reproduction in other cultures taken simultaneously with CSF (such as blood, tracheal aspirate, and urine)

Meningitis with another agent was accepted as a new case 7 days after the eradication of the first agent from CSF. After 30 days of eradication of agent, sterilization of CSF and recovery without sequelae were considered as a cure.

Demographic, clinical, and treatment applications of the cases meeting our criteria were obtained from computer and electronic file records. Causes of intervention, administered antibiotics, microorganisms isolated from CSF and their sensitivities, treatment methods, and disease outcomes were all recorded. Response to treatment was evaluated with daily patient visits, clinical course, and biochemical and microbiological examinations of CSF samples taken every 48 hours.

CSF samples for POM diagnosis were taken from lumbar puncture and ventricular or lumbar drainage catheter. CSF and blood culture were taken simultaneously and kept at room temperature while being delivered to the laboratory. All CSF samples were sown in 5\% sheep blood agar and chocolate agar (containing $\mathrm{X}$ and $\mathrm{V}$ content) and incubated at $37^{\circ} \mathrm{C}$ for 72 hours in $5 \% \mathrm{CO}_{2}$ medium. The BACTEC ${ }^{\mathrm{TM}}$ Automated Blood Culture System (Becton Dickinson) was used for blood cultures.

The standard microbiological evaluation was made on the isolates (Gram staining, colony morphology, catalase, oxidase, and coagulase tests). VITEK 2 system (bioMérieux, France) was used for bacterial identification and susceptibility.

2.1. Administration of IT/IVT Agents. As soon as the patients were diagnosed with POM, a catheter was inserted and treatment was started. Due to low CSF penetration of IV drugs and nosocomial resistant microorganisms, simultaneous IT/IVT treatment process was aimed. Antibiotherapy was revised according to culture results and antibiograms.

The IT/IVT agents amikacin and colistin were administered as described below:

(1) Amikacin $30 \mathrm{mg} /$ day and colistin $10 \mathrm{mg} /$ day were administered

(2) The administration was made through the intraventricular/lumbar catheter; firstly, $5 \mathrm{cc}$ of CSF was sampled and the catheter line was emptied

(3) All antibiotics were diluted with 5 cc $0.9 \% \mathrm{NaCl}$ and given through the catheter and the drainage was closed for 1 hour 
(4) CSF culture was taken every 48 hours

(5) Sterility of the CSF samples taken 3 times in a row was considered as cure

2.2. Statistics. Results are presented as mean \pm standard deviation and median ( $\min -\max$ ) for continuous variables and $\mathrm{n}$ (percentage) for categorical variables.

Ethics committee approval was received from Istanbul Training and Research Hospital Clinical Research Ethics Committee on 09/08/2019 (decision no. 1940).

\section{Results}

A total of 1562 neurosurgical operations were performed in Istanbul Training and Research Hospital between January 2016 and December 2019 with different indications. Postoperatively, 1023 of these cases were followed up in the ICU.

POM was detected in 18 of 1023 patients with ICU follow-up (1.75\%). Fourteen of 18 detected cases were diagnosed with MDR POM (77.7\%) (Figure 1). 71.42\% (10/14) of the cases were male and the mean age was $48.28 \pm 16.93$ years (range: 16-75 years). Operation indications were intracranial bleeding (8 cases), hydrocephalus (2 cases), traumatic brain injury ( 2 cases), intraventricular bleeding (1 case), and ventriculoperitoneal shunt revision (1 case). Extraventricular drainage (EVD) catheter (10 cases) and lumbar drainage catheter (4 cases) were placed to reduce intracranial pressure in patients. The prevalence of MDR POM was $0.9 \%$ in total.

In patients with MDR, A. baumannii, 71.43\% (10/14), and $K$. pneumoniae, $28.57 \%$, were the causative agents. The average time until POM was detected as $12.07 \pm 11.84$ days. Vancomycin ( 14 cases), meropenem ( 7 cases), ceftazidime ( 6 cases), and ceftriaxone (1 case) were used as empirical treatments. Before IT/IVT therapy, empirical antibiotics were applied for $10 \pm 8.8$ days.

At the time of diagnosis, CSF glucose was $11.93 \pm 11.83 \mathrm{~g} / \mathrm{dL}$, protein was $336.69 \pm 219.89 \mathrm{mg} / \mathrm{dL}$, and leukocyte count was $886.43 \pm 364.81 / \mathrm{mm}^{3}$. Meropenemcolistin combination was used IV in 7 of 10 patients diagnosed with $A$. baumannii, with tigecycline-colistin in 2 patients and tigecycline-colistin-vancomycin in 1 patient. While meropenem-amikacin combination was used in 3 out of 4 patients diagnosed with $K$. pneumoniae, meropenemvancomycin was used in 1 patient. Microbiological eradication was achieved in $3 \pm 0.8$ days with $30 \mathrm{mg} /$ day amikacin treatment in POM due to K. pneumoniae and $3.7 \pm 1.95$ days with colistin sodium $10 \mathrm{mg} /$ day treatment in POM due to A. baumannii via IT/IVT catheter. IT/IVT treatment was utilized for a median of 10 days and continued until the defined cure criteria were achieved. There was no complication observed related to the administration of IT/IVT amikacin and colistin.

While cure was achieved in 6 of 14 POM cases, 8 of them were exitus. Of the 6 patients who were cured, 3 died due to cerebral hemorrhage; the other 3 patients were discharged with permanent shunt. Demographic data, operation indications, prediagnosis CSF findings, and CSF findings during

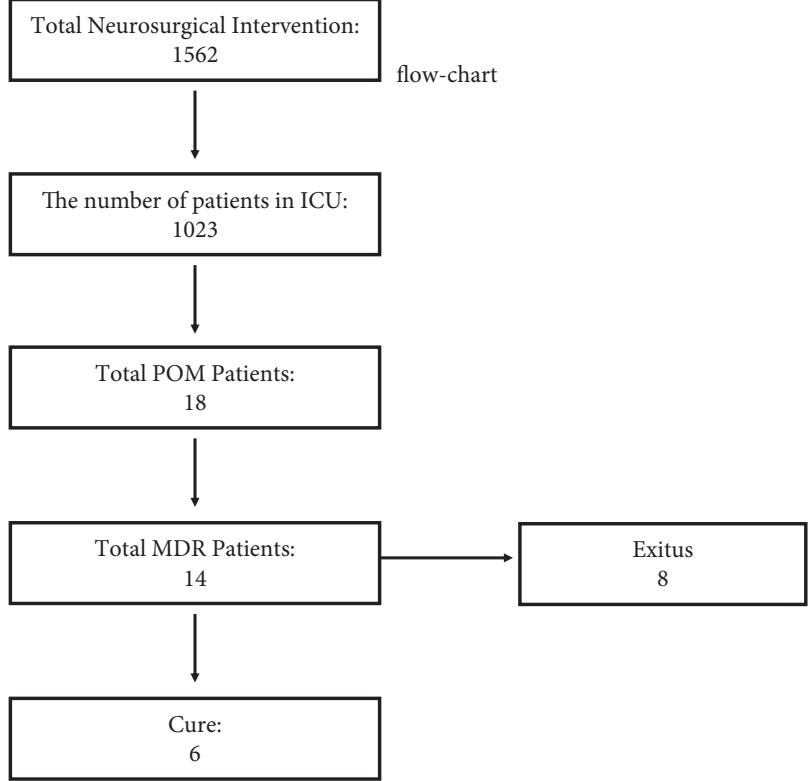

FIGURE 1: Patient flowchart.

the period when microbiological eradication was achieved, as well as empirical and antibiotic treatments against the agent, are detailed in Table 1.

\section{Discussion}

The exact diagnosis of POM is made with a combination of clinical signs plus microbiological and biochemical analysis of the CSF. Gram-negative bacteria are responsible for $60-70 \%$ of POMs. A. baumannii has an important place among Gram-negative bacteria. Long-term use of broadspectrum and inappropriate antibiotics, use of ventricular catheters for more than 5 days, and ventriculostomy are risk factors for A. baumannii meningitis [15-22]. While the mortality rate in meningitis caused by $A$. baumannii varies between 30 and $50 \%$, this rate is reported to be as high as $50-70 \%$ in MDR A. baumannii meningitis [23]. In our study, prevalence of POM related to A. baumannii was $71.43 \%$ and $60 \%$ of our cases lost their lives.

In a study conducted in Turkey in 2020, Gram-negative meningitis cases treated with IT/IVT were examined [24]. The most common microorganism in 13 patients included in the study was $A$. baumannii (8/13). Colistin was used consecutively in 8 patients, amikacin in 4 patients, and amikacin plus colistin in 1 patient. The 28-day infectionrelated mortality was $15 \%$ with 2 patients. In our study, mortality was $57.1 \%$ with 8 patients in a total of $14 \mathrm{MDR}$ POM cases. In patients with MDR, A. baumannii in $71.43 \%$ $(10 / 14)$ and $K$. pneumoniae in $28.57 \%$ of patients were the causative agents. The most commonly used antimicrobial agent was colistin.

Another study in 2019 [25] examined a total of 25 POM cases with XDR A. baumannii infection. All patients received IVT colistin and the mean duration of colistin usage was $13.4 \pm 2.8$ days. The time to obtain a negative CSF culture was $8.9 \pm 4.0$ days. In this study, mortality was $20 \%$ 
TABLE 1: Demographic data, operation indications, microbiological agent, empirical and agent targeted antibiotic preferences, and durations of therapies.

\begin{tabular}{|c|c|c|c|c|c|c|c|c|c|c|}
\hline Case & $\begin{array}{l}\text { Age/ } \\
\text { gender }\end{array}$ & $\begin{array}{l}\text { Operation } \\
\text { indication }\end{array}$ & $\begin{array}{c}\text { Days of POM } \\
\text { development }\end{array}$ & $\begin{array}{l}\text { Empirical } \\
\text { therapy }\end{array}$ & $\begin{array}{c}\text { Microbiological } \\
\text { agent }\end{array}$ & $\begin{array}{c}\text { Agent } \\
\text { targeted } \\
\text { therapy } \\
\text { (IV) }\end{array}$ & $\begin{array}{l}\text { Duration } \\
\text { (days) }\end{array}$ & $\begin{array}{l}\text { IV/IVT } \\
\text { therapy }\end{array}$ & $\begin{array}{l}\text { Duration } \\
\text { (days) }\end{array}$ & $\begin{array}{l}\text { Last } \\
\text { status }\end{array}$ \\
\hline 1 & $52 / \mathrm{M}$ & $\begin{array}{c}\text { Intraventricular } \\
\text { hemorrhage }\end{array}$ & 12 & Mem, Va & A. baumanni & Mem, Col & 5 & $\mathrm{Col}$ & 5 & Exitus \\
\hline 2 & $56 / \mathrm{M}$ & Hydrocephalus & 1 & $\mathrm{Caz}, \mathrm{Va}$ & A. baumanni & Tig, Col & 21 & Col & 10 & Cure \\
\hline 3 & $54 / \mathrm{M}$ & Aneurysm & 11 & $\mathrm{Caz}, \mathrm{Va}$ & A. baumanni & Mem, Col & 5 & $\mathrm{Col}$ & 5 & Exitus \\
\hline 4 & $54 / \mathrm{M}$ & Hydrocephalus & 34 & $\mathrm{Caz}, \mathrm{Va}$ & K. pneumoniae & Mem, Ak & 15 & $\mathrm{Ak}$ & 10 & Cure \\
\hline 5 & $39 / \mathrm{F}$ & Aneurysm & 9 & $\mathrm{Mem}, \mathrm{Va}$ & A. baumanni & Mem, Col & 7 & Col & 7 & Exitus \\
\hline 6 & $42 / \mathrm{M}$ & Aneurysm & 15 & $\mathrm{Caz}, \mathrm{Va}$ & A. baumanni & Mem, Col & 12 & $\mathrm{Col}$ & 7 & Exitus \\
\hline 7 & $16 / \mathrm{M}$ & Aneurysm & 7 & $\mathrm{Mem}, \mathrm{Va}$ & A. baumanni & Tig, Col & 20 & Col & 14 & Cure \\
\hline 8 & $49 / \mathrm{M}$ & Aneurysm & 10 & Caz, Va & A. baumanni & $\begin{array}{l}\text { Tig, Va, } \\
\text { Col }\end{array}$ & 20 & Col & 14 & Cure \\
\hline 9 & $71 / \mathrm{M}$ & Aneurysm & 42 & $\mathrm{Caz}, \mathrm{Va}$ & A. baumanni & Mem, Col & 14 & $\mathrm{Col}$ & 10 & Cure \\
\hline 10 & $16 / \mathrm{M}$ & Shunt revision & 2 & $\mathrm{Mem}, \mathrm{Va}$ & K. pneumoniae & Mem, Ak & 13 & $\mathrm{Ak}$ & 10 & Exitus \\
\hline 11 & $42 / \mathrm{F}$ & Trauma & 6 & Cro, Va & A. baumanni & Mem, Col & 12 & $\mathrm{Col}$ & 10 & Exitus \\
\hline 12 & $52 / \mathrm{M}$ & Aneurysm & 12 & Mem, Va & A. baumanni & Mem, Col & 10 & $\mathrm{Col}$ & 10 & Exitus \\
\hline 13 & $75 / \mathrm{M}$ & Trauma & 2 & $\mathrm{Mem}, \mathrm{Va}$ & K. pneumoniae & Mem, Va & 21 & $\mathrm{Ak}$ & 10 & Cure \\
\hline 14 & $58 / \mathrm{F}$ & Aneurysm & 6 & Mem, Va & K. pneumoniae & Mem, Ak & 7 & $\mathrm{Ak}$ & 7 & Exitus \\
\hline
\end{tabular}

IV: intravenous; IVT: intraventricular; Mem: meropenem; Va: vancomycin; Caz: ceftazidime; Cro: ceftriaxone; Tig: tigecycline; Col: colistimethate sodium; Ak: amikacin.

with 5 patients. In another study from 2018 [26], a retrospective cohort was conducted in patients who were diagnosed with POM and admitted to ICU receiving IVT antibiotic therapy. All 105 patients enrolled in the study received systemic antimicrobial therapy in combination with at least one dose of an IVT antimicrobial agent. IVT vancomycin was used in $52.4 \%$ of the patients. Overall mortality was $18.1 \%$ with 19 patients. CSF culture sterilization was achieved in $88.4 \%$ of patients. In another study in 2018 [27], a total of 61 cases of POM infected with MDR microbial agents were examined. The rate of MDR A. baumannii infection was $33.64 \%$. The patients were treated with polymyxin B. Compared to the IV treatment group, the IT/ IVT treatment group had lower 28-day mortality (55.26\% and $8.70 \%, p=0.01)$ and higher rates of clinical and antimicrobiological efficacy (95.65\% versus $23.68 \%, p<0.001 \%$; $91.30 \%$ versus $18.42 \%, p<0.001$ ), respectively. In our study, microbiological eradication was achieved in $3 \pm 0.8$ days with $30 \mathrm{mg} /$ day amikacin treatment in POM due to K. pneumoniae and $3.7 \pm 1.95$ days with colistin $10 \mathrm{mg}$ /day treatment in POM due to A. baumannii via IT/IVT catheter. Cure was achieved in 6 of 14 POM cases and 8 of them were exitus.

The fact that A. baumannii is increasingly resistant to many antibiotics including carbapenem group is an important problem in treatment in healthcare-related infections [28]. Tuon et al. detected carbapenem resistance in $40.9 \%$ of nosocomial meningitis. In addition, they identified the most important cause of mortality as the inappropriate administration of antibiotic therapy within five days of receiving CSF culture [18]. In our study, all A. baumannii strains were resistant to carbapenem and the empirical treatments applied were insufficient.
At this point, it would be good to mention ceftazidime/ avibactam. Both the coverage of carbapenem-resistant Gram-negative bacteria and the better CSF penetration compared to other beta-lactam antibiotics cause significant advantages [11]; however, since it is not in use in our country, it is not included in our study.

As a result of blood-brain barrier impairment and alteration of drug pharmacokinetics due to CNS infections, intravenously administered drugs could not reach the desired effective concentrations. IT/IVT administration is generally preferred in the presence of resistant microorganisms, where systemic IV antibiotic therapy is thought to be inadequate. The main reason why these methods are not preferred in the first place are dose-dependent toxic reactions such as seizure, chemical ventriculitis, and transient hearing loss (after gentamicin and vancomycin) shown in previous studies [29-31]. On the other hand, there are some studies reporting that the side effects related to polymyxin B, colistimethate sodium, and vancomycin are negligible and that these minimal side effects are also dose-dependent [32-34].

Rodriquez et al. compared different routes of antibiotic administration in nosocomial A. baumannii infection and reported that both intraventricular and intrathecal colistin treatments are useful and safe options [22]. It was reported that colistin, gentamicin, amikacin, and polymyxin $B$ antibiotics can be used in the treatment of resistant $A$. baumannii strains via IT/IVT [35]. In a metaanalysis in 2018 [36], 11 studies with 348 patients met eligibility criteria. In this study comparing IVT plus IV treatment and IV treatment, IVT plus IV treatment was statistically superior to IV therapy alone in eradication and mortality. 
Remes et al. firstly reported that, alternatively, meropenem and netilmicin therapy will also be effective without observing side effects [33]. In a study of 21 patients, Khan et al. reported that IT/IVT antibiotic administration is beneficial and has minimal side effects in cases of postoperative Gram-negative meningitis and ventriculitis that do not respond to standard therapy [34]. In our retrospective study, no complication was observed with the administration of IT/IVT amikacin and colistin.

In our small series study, doses of the administered $\operatorname{drug}(\mathrm{s})$ and clinical data of the patients could not be compared with detailed statistical methods, as the retrospective study was insufficient in the clinical determination of drug side effects in the cases that were followed up under sedation in the intensive care unit.

\section{Conclusion}

As a result, we believe that, in selected cases, to avoid the severe consequences of postoperative meningitis, acting fast and adding IT/IVT methods to parenteral administration routes by considering the distribution of MDR microorganisms within the hospital while planning effective treatment will increase the clinical success.

\section{Abbreviations}

$\begin{array}{ll}\text { POM: } & \text { Postoperative meningitis } \\ \text { S. aureus: } & \text { Staphylococcus aureus } \\ \text { P. acnes: } & \text { Propionibacterium acnes } \\ \text { K. pneumoniae: } & \text { Klebsiella pneumoniae } \\ \text { P. aeruginosa: } & \text { Pseudomonas aeruginosa } \\ \text { XDR: } & \text { Extensively drug-resistant (XDR) } \\ \text { MDR: } & \text { Multidrug-resistant } \\ \text { ICU: } & \text { Intensive care unit } \\ \text { CSF: } & \text { Cerebrospinal fluid } \\ \text { NM: } & \text { Nosocomial meningitis } \\ \text { CDC: } & \text { American Centers for Disease Control and } \\ & \text { Prevention } \\ \text { EVD: } & \text { Extraventricular drainage } \\ \text { IVT: } & \text { Intraventricular } \\ \text { IT: } & \text { Intrathecal. }\end{array}$

\section{Data Availability}

The clinical data used to support the findings of this study are available from the corresponding author upon request.

\section{Ethical Approval}

Ethical committee approval was received (approval date and number: 09/08/2019/1940). The experimental procedures were based on the Declaration of Helsinki and relevant institutional regulations.

\section{Consent}

An informed consent was obtained as written forms from all of our patients before beginning the study for publication.

\section{Conflicts of Interest}

The authors declare that they have no conflicts of interest.

\section{Authors' Contributions}

All authors contributed to the editing of the manuscript. IS wrote the manuscript and made the accompanying tables.

\section{References}

[1] S. L. Berk and W. R. McCabe, "Meningitis caused by gramnegative bacilli," Annals of Internal Medicine, vol. 93, no. 2, pp. 253-260, 1980.

[2] C. Logigan, D. Mihalache, and T. Turcu, "Clinical study of 57 cases of nosocomial meningitis," American Journal of Preventive Medicine, vol. 16, pp. 59-68, 2008.

[3] J. Mancebo, P. Doming, L. Blanch, P. Coll, A. Net, and J. Nolla, "Post-neurosurgical and spontaneous gram-negative bacillary meningitis in adults," Scandinavian Journal of Infectious Diseases, vol. 18, no. 6, pp. 533-538, 1986.

[4] K. Hussein, R. Bitterman, B. Shofty, M. Paul, and A. Neuberger, "Management of post-neurosurgical meningitis: narrative review," Clinical Microbiology and Infections, vol. 23, no. 9, pp. 621-628, 2017.

[5] B. Kurtaran, F. Kuscu, A. Ulu et al., "The causes of postoperative meningitis: the comparison of gram-negative and gram-positive pathogens," Turkish Neurosurgery, vol. 28, no. 4, pp. 589-596, 2018.

[6] M. Weisfelt, D. van de Beek, L. Spanjaard, and J. de Gans, "Nosocomial bacterial meningitis in adults: a prospective series of 50 cases," Journal of Hospital Infection, vol. 66, no. 1, pp. 71-78, 2007.

[7] B. Ceylan, F. Arslan, O. R. Sipahi et al., "Variables determining mortality in patients with Acinetobacter baumannii meningitis/ventriculitis treated with intrathecal colistin," Clinical Neurology and Neurosurgery, vol. 153, pp. 43-49, 2017.

[8] I. Karaiskos, L. Galani, F. Baziaka et al., "Successful treatment of extensively drug-resistant Acinetobacter baumannii ventriculitis and meningitis with intraventricular colistin after application of a loading dose: a case series," International Journal of Antimicrobial Agents, vol. 41, no. 5, pp. 480-483, 2013.

[9] R. Dersch, E. Robinson, L. Beume, S. Rauer, and W.-D. Niesen, "Full remission in a patient with catheterassociated ventriculitis due to Acinetobacter baumannii treated with intrathecal and intravenous colistin besides coinfections with other multidrug-resistant bacteria," Neurological Sciences, vol. 36, no. 4, pp. 633-634, 2015.

[10] Y. Hu, W. He, D. Yao, and H. Dai, "Intrathecal or intraventricular antimicrobial therapy for post-neurosurgical intracranial infection due to multidrug-resistant and extensively drug-resistant gram-negative bacteria: a systematic review and meta-analysis," International Journal of Antimicrobial Agents, vol. 54, no. 5, pp. 556-561, 2019.

[11] V. Pomar, N. Benito, J. López-Contreras, P. Coll, M. Gurguí, and P. Domingo, "Spontaneous gram-negative bacillary meningitis in adult patients: characteristics and outcome," BMC Infectious Diseases, vol. 13, no. 1, p. 451, 2013.

[12] I. Karaiskos, L. Galani, F. Baziaka, and H. Giamarellou, "Intraventricular and intrathecal colistin as the last therapeutic resort for the treatment of multidrug-resistant and extensively drug-resistant Acinetobacter baumannii ventriculitis and meningitis: a literature review," International 
Journal of Antimicrobial Agents, vol. 41, no. 6, pp. 499-508, 2013.

[13] A.-P. Magiorakos, A. Srinivasan, R. B. Carey et al., "Multidrug-resistant, extensively drug-resistant and pandrug-resistant bacteria: an international expert proposal for interim standard definitions for acquired resistance," Clinical Microbiology and Infections, vol. 18, no. 3, pp. 268-281, 2012.

[14] T. C. Horan, M. Andrus, and M. A. Dudeck, "CDC/NHSN surveillance definition of health care-associated infection and criteria for specific types of infections in the acute care setting," American Journal of Infection Control, vol. 36, no. 5, pp. 309-332, 2008.

[15] G. Metan, E. Alp, B. Aygen, and B. Sumerkan, "Carbapenemresistant Acinetobacter baumannii: an emerging threat for patients with post-neurosurgical meningitis," International Journal of Antimicrobial Agents, vol. 29, no. 1, pp. 112-113, 2007.

[16] L. L. Maragakis and T. M. Perl, "Antimicrobial resistance: acinetobacter baumannii: epidemiology, antimicrobial resistance, and treatment options," Clinical Infectious Diseases, vol. 46, no. 8, pp. 1254-1263, 2008.

[17] J. Garnacho-Montero and R. Amaya-Villar, "Multiresistant Acinetobacter baumannii infections: epidemiology and management," Current Opinion in Infectious Diseases, vol. 23, no. 4, pp. 332-339, 2010.

[18] F. F. Tuon, S. R. Penteado-Filho, D. Amarante, M. A. Andrade, and L. A. Borba, "Mortality rate in patients with nosocomial Acinetobacter meningitis from a Brazilian hospital," Brazilian Journal of Infectious Diseases, vol. 14, no. 5, pp. 437-440, 2010.

[19] M. E. Jiménez-Mejías, J. Pachón, B. Becerril, J. PalominoNicás, A. Rodríguez-Cobacho, and M. Revuelta, "Treatment of multidrug-resistant Acinetobacter baumannii meningitis with ampicillin/sulbactam," Clinical Infectious Diseases, vol. 24, no. 5, pp. 932-935, 1997.

[20] S. Laxmi and A. R. Tunkel, "Healthcare-associated bacterial meningitis," Current Infectious Disease Reports, vol. 13, no. 4, pp. 367-373, 2011.

[21] A. Cascio, A. Conti, L. Sinardi et al., "Post-neurosurgical multidrug-resistant Acinetobacter baumannii meningitis successfully treated with intrathecal colistin. a new case and a systematic review of the literature," International Journal of Infectious Diseases, vol. 14, no. 7, pp. e572-e579, 2010.

[22] A. Rodríguez Guardado, A. Blanco, V. Asensi et al., "Multidrug-resistant Acinetobacter meningitis in neurosurgical patients with intraventricular catheters: assessment of different treatments," Journal of Antimicrobial Chemotherapy, vol. 61, no. 4, pp. 908-913, 2008.

[23] S. McClelland and W. A. Hall, "Postoperative central nervous system infection: incidence and associated factors in 2111 neurosurgical procedures," Clinical Infectious Diseases, vol. 45, no. 1, pp. 55-59, 2007.

[24] M. Ayhan, A. Kaya Kalem, I. Hasanoglu et al., "Intrathecal and intraventricular administration of antibiotics in gramnegative nosocomial meningitis in a research hospital in Turkey," Turkish Neurosurgery, vol. 31, no. 3, pp. 348-354, 2021.

[25] F. Chen, X. Deng, Z. Wang, L. Wang, K. Wang, and L. Gao, "Treatment of severe ventriculitis caused by extensively drugresistant Acinetobacter baumannii by intraventricular lavage and administration of colistin," Infection and Drug Resistance, vol. 12, pp. 241-247, 2019.

[26] J. J. Lewin, A. M. Cook, C. Gonzales et al., "Current practices of intraventricular antibiotic therapy in the treatment of meningitis and ventriculitis: results from a multicenter retrospective cohort study," Neurocritical Care, vol. 30, no. 3, pp. 609-616, 2019.

[27] S. Pan, X. Huang, Y. Wang et al., "Efficacy of intravenous plus intrathecal/intracerebral ventricle injection of polymyxin $\mathrm{B}$ for post-neurosurgical intracranial infections due to MDR/ XDR Acinectobacter baumannii: a retrospective cohort study," Antimicrobial Resistance \& Infection Control, vol. 7, no. 1, p. 8, 2018.

[28] A. Camkıran, A. Kundakcı, Ç. Araz, A. Pirat, P. Zeyneloğlu, and H. Arslan, "Preliminary determinants of multidrug-resistant Acinetobacter baumannii infection in the surgical intensive care unit: a retrospective analysis," Journal of Turkish Intensive Care Association, vol. 9, pp. 53-58, 2011.

[29] T. Khawcharoenporn, A. Apisarnthanarak, and L. M. Mundy, "Intrathecal colistin for drug-resistant Acinetobacter baumannii central nervous system infection: a case series and systematic review," Clinical Microbiology and Infections, vol. 16, no. 7, pp. 888-894, 2010.

[30] S. Ni, S. Li, N. Yang et al., "Post-neurosurgical meningitis caused by acinetobacter baumannii: case series and review of the literature," International Journal of Clinical and Experimental Medicine, vol. 8, no. 11, pp. 21833-21838, 2015.

[31] B. A. Dorum, T. Bal, U. Ozer, F. D. Aygun, and İ. Şilfeler, "İntraventrikuler kolistin tedavisine bağlı gelişen kimyasal ventrikulit olgusu," Journal of Clinical and Analytical Medicine, vol. 5, no. Suppl. 4, pp. 524-526, 2014.

[32] W. C. Ziai and J. J. Lewin, "Improving the role of intraventricular antimicrobial agents in the management of meningitis," Current Opinion in Neurology, vol. 22, no. 3, pp. 277-282, 2009.

[33] F. Remeš, R. Tomáš, V. Jindrák, V. Vaniš, and M. Šetlík, "Intraventricular and lumbar intrathecal administration of antibiotics in postneurosurgical patients with meningitis and/ or ventriculitis in a serious clinical state," Journal of Neurosurgery, vol. 119, no. 6, pp. 1596-1602, 2013.

[34] M. Shamim, S. Khan, M. Waqas et al., "Intrathecal and intraventricular antibiotics for postoperative gram-negative meningitis and ventriculitis," Surgical Neurology International, vol. 8, no. 1, p. 226, 2017.

[35] A. R. Tunkel, R. Hasbun, A. Bhimraj et al., "2017 infectious diseases society of America's clinical practice guidelines for healthcare-associated ventriculitis and meningitis," Clinical Infectious Diseases, vol. 64, no. 6, pp. e34-e65, 2017.

[36] M. Karvouniaris, A. G. Brotis, P. Tsiamalou, and K. N. Fountas, "The role of intraventricular antibiotics in the treatment of nosocomial ventriculitis/meningitis from gramnegative pathogens: a systematic review and meta-analysis," World Neurosurgery, vol. 120, pp. e637-e650, 2018. 